\title{
Regional Risk Assessment of Earthquake-triggered Landslides
}

\author{
Yingying Tian \\ Key Laboratory of Active Tectonics and Volcano, Institute of Geology, China Earthquake Administration, Beijing \\ 100029, China \\ School of Engineering and Technology, China University of Geosciences (Beijing), Beijing 100083, China \\ E-mail:t190403098@163.com \\ Chong Xu \\ Key Laboratory of Active Tectonics and Volcano, Institute of Geology, China Earthquake Administration, Beijing \\ 100029, China \\ E-mail: xuchong@ies.ac.cn \\ Jian Chen \\ School of Engineering and Technology, ChinaUniversity of Geosciences (Beijing), Beijing 100083, China \\ E-mail:jianchen@cugb.edu.cn
}

Received 10 June 2015

Accepted 26 October 2015

\begin{abstract}
Great earthquakes occurring in mountainous areas can trigger large-scale landslides, leading to serious geological disasters. Thus, in recent years, especially after the 2008 Wenchuan earthquake, much attention has been focused on the research about regional risk assessment of seismic landslides in China and elsewhere in the world. Such study is based on the engineering geological analogy, and its purpose is to estimate the risks of earthquake-induced landslides for the regions with the same or similar environment. In light of previous work, such assessment includes 2 tasks: establishment of seismic landslide database and evaluation of potential landslides using mathematical statistics models. The interpretation of regional seismic landslides is the basis for building the landslide database. The common methods for risk assessment include the evidence-weight model, certainty factor method (CF)and information value model, logistic regression model (LR), artificial neural networks (ANN), support vector machine method (SVM), Newmark displacement model,analytic hierarchy process (AHP), and so forth. This paper presents a review on these methods, and an outlook on the advancement of this research field in the future.
\end{abstract}

Keywords:Seismic, Landslide, Hazard assessment. 


\title{
区域地震滑坡危险性评价
}

\author{
田颖颙 \\ 中国地震局地质研究所, 活动构造与火山重点实验室, 北京 100029 \\ 中国地质大学 (北京)，工程技术学院，北京 100083 \\ E-mail:t190403098@163.com \\ 许冲 \\ 中国地震局地质研究所, 活动构造与火山重点实验室, 北京 100029 \\ E-mail:xuchong@ies.ac.cn \\ 陈剑 \\ 中国地质大学 (北京)，工程技术学院，北京 100083 \\ E-mail:jianchen@cugb.edu.cn
}

\begin{abstract}
摘要
山区大地震通常触发大规模滑坡并诱发严重的地质灾害。近年来，特别是 2008 年汶川地震之后，国内 学者高度重视区域地震滑坡危险性评价研究。它以工程地质类比法为理论基础, 去估计具有相同或类似环 境条件的区域尺度地震滑坡的危险性。从前人工作来看, 区域危险性评价主要包括 2 方面: 建立地震滑坡 数据库和运用数学统计模型进行危险性评价。数据库建立以区域地震滑坡解译为基础, 地震滑坡危险性评 价常用方法包括: 证据权重模型、确定性系数法 $(C F)$ 、信息量法、逻辑回归模型 $(L R) 、$ 人工神经网络 $(A N N)$ 、 支持向量机法 $(S V M)$ 、Newmark 位移模型和层次分析法 $(A H P)$ 等。本文对各种评价方法做了综述，并对 地震滑坡危险评价研究趋势提出了一些看法。
\end{abstract}

关键词：地震；滑坡；危险性评价

\section{1. 引言}

地震滑坡泛指由地震所诱发的次生地质灾害, 包 括崩、滑、流等。在地震发生之后，地震滑坡波及面 积之广、发生频率之高、运动速度之快、灾害损失之 严重决定其致灾能力不亚于地震本身, 因此引起了广 泛关注。早在公元前 1789 年中国和公元前 373 年或 372 年希腊就已经有了地震滑坡的记录, 第一次正式 被用来进行科学研究的大地震为 1783 年发生在意大 利的 Calabria 地震（Keefer, 2002）。近年来国内外高 震级地震频发, 地震及其诱发的次生地质灾害造成了 严重的人员伤亡和经济损失。1920 年 12 月 16 日宁 夏海原 Ms 8.5 级地震, 诱发了至少 805 处大型黄土滑 坡, 其所占的面积约 5 万 $\mathrm{km}^{2}$ (吴玲, 2012; Li 等, 2013b）。1994 年 1 月 17 日美国加州的 Northridge Mw6.7 级地震在 1 万 $\mathrm{km}^{2}$ 的面积内触发了至少 11,000
处滑坡, 且滑坡的面积多大于 $1000 \mathrm{~km}^{2}$, 造成了重大 的损失（Harp and Jibson, 1996）。1999 年 9 月 21 日台 湾 Ms7.6 级集集地震, 引发 13,175 处滑坡, 造成了重 大的经济损失 (Lee, 2013)。2008 年 5 月 12 日四川 汶川 Ms8.0 级地震诱发了 197,481 处滑坡灾害, 直接 造成了 2 万人死亡和巨大的经济损失 (殷跃平, 2009)。 2010 年 1 月 12 日海地太子港 Mw7.0 级地震触发了至 少 30,828 处滑坡, 造成 23 万多人遇难和近 80 亿美元 的经济损失（Calais 等, 2010; 许冲, 2013a)。2010 年 4 月 14 日青海玉树 Ms7.1 级地震在面积约 $1455.3 \mathrm{~km}^{2}$ 的矩形区域内诱发了 2,036 处滑坡, 造成的直接经济 损失约 60 万元（许冲等, 2011）。2013 年 4 月 20 日芦 山 Ms7.0 级地震解译出滑坡 3,883 处, 累计受灾人口 达 38.3 万人（许冲, 2013b)。2013 年 7 月 22 日甘肃 岷县漳县 Ms 6.6 级地震中仅“永光村 1 \#滑坡” 滑坡 (体积约 $4.2 \times 10^{4} \mathrm{~m}^{3}$ ) 和“永光村 2 \#滑坡”滑坡（体积 
约 $\left.3.3 \times 10^{4} \mathrm{~m}^{3}\right)$, 就造成了永光村 4 社瞬间被夷为废 墟, 造成了至少 12 人遇难（许冲等, 2013）。鉴于地 震滑坡的巨大致灾能力, 地震滑坡灾害危险性评价显 得尤为重要。

\section{2. 地震滑坡危险性评价概述}

进行地震滑坡危险性评价主要有两种思路: 一是 对大型单体地震滑坡进行物理力学性质分析, 并用力 学模型去模拟滑坡发生的环境, 达到危险性评价的目 的; 二是基于地震、地质和地形地貌等影响地震滑坡 发生的因素, 对区域性地震滑坡进行统计分析。本文 主要针对区域性地震滑坡的危险性评价方法进行论 述。

区域性地震滑坡危险性评价利用工程地质类比 法思想，根据地震后已经发生滑坡的空间分布信息反 演地震滑坡涉及范围内的滑坡易发条件, 从而进行危 险性区划, 为以后相似地质环境下的防灾减灾工作提 供参考。影响地震滑坡的因素有很多, 包括地震因素、 地质因素和地形地貌因素, 由于这些因素具有不确定 性和模糊性, 加之有些因素很难获得或量化, 因此地 震滑坡危险性评价十分复杂, 目前还没有成熟的方法 (陈晓利等, 2008; 庄建琦等, 2010)。

早期人们对地震滑坡研究主要依靠实地调查。20 世纪 30 至 40 年代, 航空照片的出现, 成为了记录地 震滑坡发生的重要手段。航空照片使得我们可以很方 便地辨别滑坡的位置以及规模, 大大减少了野外工作 量, 为滑坡的编目提供了较为便捷的途径。20 世纪 80 年代高精度卫星影像的出现, 进一步推动了地震滑 坡数据库的建立 (Keefer, 2002)。同时随着 GIS 的出 现，地震滑坡危险性评价过程中复杂空间分析的需求
得到了很好地解决, 进一步刺激了地震滑坡危险性评 价的发展。

通过野外实地调查和各种遥感数据解译可以获 得客观、详细、完整的滑坡编目; 然后结合地震、地 质及地形资料, 进而获得地震滑坡的空间分布规律。 最后借助各种数学模型进行滑坡危险性评价。进行地 震滑坡危险性评价时常常参考研究比较成熟的降雨 诱发滑坡危险性评价的研究成果（葛华等, 2013)。

\section{3. 地震滑坡危险性评价的步骤}

地震滑坡危险性评价的步骤可简要概括为:

(1) 进行滑坡编目。详细精确的滑坡编目是地 震滑坡危险性分析工作中必不可少的一部分 (Harp 等, 2011; 许冲和徐锡伟, 2014; Xu, 2015)。借助连续且覆 盖率高的高分辨率遥感影像, 用点要素或面要素去标 识滑坡, 圈定出滑坡的轮廓和位置, 并对特殊的滑坡 灾害进行现场调查和核实, 创建地震滑坡数据库, 这 是进行地震滑坡危险性评价的基础。尤其是 2008 年 汶川地震之后, 出现了多个基于地震事件的同震滑坡 数据库成果, 如 2010 年甘肃玉树地震 ( $\mathrm{Xu}$ and $\mathrm{Xu}$, 2014)、2010 年海地地震（Xu et al., 2014a)、2013 年 四川芦山地震 (Xu et al., 2015a; Xu et al., 2015b)、2013 年甘肃岷县地震 (Xu et al., 2014b)、2014 年云南鲁甸 地震（许冲 et al., 2014）等。其中 2008 年四川汶川 地震同震滑坡数据库是记录最详细、滑坡数量最多的 (Xu et al., 2014c)。

（2）开展地震滑坡危险性评价。选取影响地震 滑坡发生的地震、地质、地形地貌等因素, 基于 GIS 和各种评价模型进行地震滑坡危险性评价, 并制作危 险性区划图。

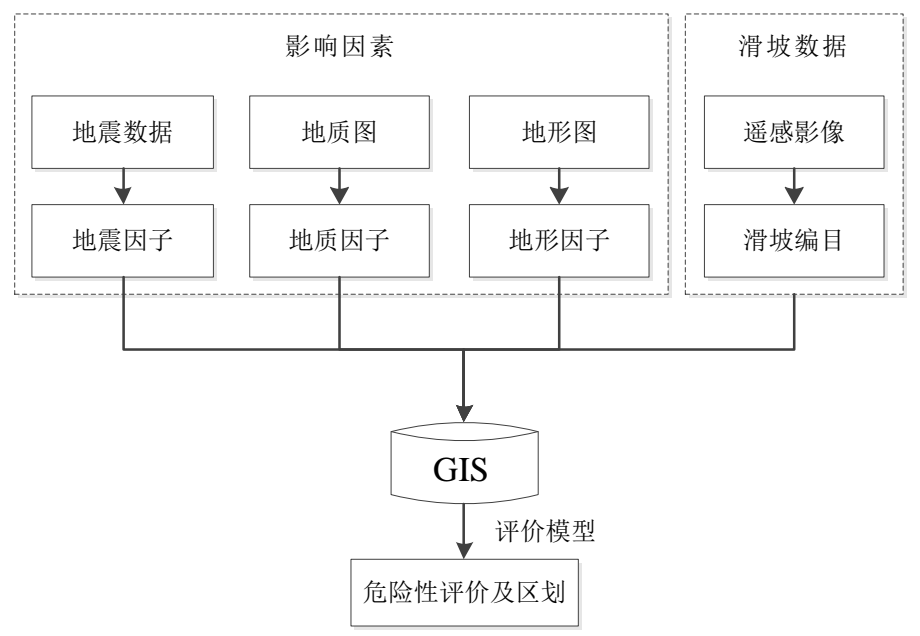

图 1.地震滑坡危险性评价流程

Fig. 1.The steps of earthquake-induced landslide hazard assessment. 


\section{4. 地震滑坡危险性评价的方法}

通过总结国内外研究者针对区域性地震滑坡危 险性评价所做的工作，危险性评价的方法大致可分为 2 大类:（1）数据驱动方法，通过研究已发生的真实 滑坡分布数据, 统计各影响因子对地震滑坡的贡献权 重, 从而得到地震滑坡危险性评价结果;（2）知识驱 动方法, 主要通过专家知识或者机器学习事件发生的 规律, 为各影响因子赋权重, 从而判断滑坡在研究区 内的分布规律（谢洪斌等, 2011; 许冲, 2014）。

常用的评价方法主要有证据权重模型、确定性系 数法 $(C F)$ 、信息量法、逻辑回归模型 $(L R)$ 、人工 神经网络 $(A N N)$ 、支持向量机模型 $(S V M)$ 、Newmark 位移模型以及层次分析 $(A H P)$ 法等。

\section{1.数据驱动方法}

\subsection{1. 证据权重模型}

证据权重模型属于数据驱动方法，是一种离散的 统计方法，其实质是根据已发生的滑坡事件来推测在 什么情况下较容易发生滑坡。它是以贝叶斯概率统计 为基础，假设各影响滑坡发生的因子证据层之间相互 独立，通过计算已有滑坡事件发生时的先验概率以及 各影响因子对滑坡发生与否的贡献值（权重），然后 基于卡方（ $\chi^{2}$ ) 检验，对两两因子证据层进行条件 独立性检验, 笁选出相关性较小的因子组合, 并计算 滑坡危险度的大小（后验概率），进而得到滑坡危险 性分布图 (Lee 和 Choi, 2004; 王志旺等, 2007; Dahal et al., 2008; 赵艳南和牛瑞卿, 2010)。

$$
\begin{aligned}
& w_{i}^{+}=\ln \frac{P\{F \mid L\}}{P\{F \mid \bar{L}\}} . \\
& w_{i}^{-}=\ln \frac{P\{\bar{F} \mid L\}}{P\{\bar{F} \mid \bar{L}\}} . \\
& w_{f}=\left(w_{i}^{+}-w_{i}^{-}\right) .
\end{aligned}
$$

其中, $P\{F \mid L\} / P\{F \mid \bar{L}\}$ 代表滑坡发生的充分率; $P\{\bar{F} \mid L\} / P\{\bar{F} \mid \bar{L}\}$ 代表滑坡发生的必要率; $w_{i}^{+}$表示 当前影响因子级别发生滑坡的概率, $w_{i}^{-}$代表影响因 子级别以外的部分发生滑坡的概率，二者的差值代表 该影响因子级别发生滑坡的权重, 即 $w_{f}$ 。可以用 $w_{f}$ 衡量影响因子对滑坡的重要性, 当 $w_{f}$ 为正, 表示该
影响因子级别有利于发生滑坡，为负表示不易发生滑 坡, 为 0 表示该影响因子级别对滑坡影响较小。

证据权重模型由于条件独立的约束, 忽略了各评 价因子之间的相互作用，但是避免了因子权重赋值的 人为主观性。正负权重值的引入又兼顾了滑坡区域和 不滑区域权重, 其评价结果也具有很高的参考价值。

谢洪斌等 (2011) 将模糊概率和条件模糊概率引 入证据权模型对 2008 年汶川地震震区岷江流域雁门 乡至映秀段进行了地震滑坡危险度区划，认为 $80 \%$ 以上的滑坡位于高和极高危险区。Xu 等 (2012b)、

$\mathrm{Xu}$ 等 (2012c) 基于证据权法分别对 2008 年汶川地 震震区清水河流域的 2321 处滑坡和 2010 年玉树地震 诱发的 2036 处滑坡进行了危险性区划, 得到模型的 成功率分别为 $71.82 \%$ 和 $80 \%$ 。张艳玲等 (2012) 利 用证据权法完成了 2008 年汶川地震后 12 个极重灾县 市的滑坡危险性分区，模型正确率达 $81 \%$ 。

\subsection{2. 确定性系数法}

确定性系数法（Centainty Factor, $C F$ ) 是一种二 元统计方法, $C F$ 由 Shortliffe 和 Buchanan 于 1975 年 提出, 后经 Heckerman (1986) 改进的一个概率函数, 利用工程地质类比思想，在假定可以根据已发生的地 震滑坡和其诱发因素之间的统计关系进行滑坡危险 性评价的基础上, 来分析各影响因素对滑坡事件发生 的敏感程度（兰恒星等, 2002, 2003）。该方法也完全 基于实际滑坡数据, 避免了不滑样本选取的随意性, 对于大区域地震滑坡危险性评价来说具有较强的适 用性。

$C F$ 函数具体表示为:

$$
C F=\left\{\begin{array}{l}
\frac{P P_{a}-P P_{s}}{P P_{a}\left(1-P P_{s}\right)}, P P_{a} \geq P P_{s} \\
\frac{P P_{a}-P P_{s}}{P P_{s}\left(1-P P_{a}\right)}, P P_{a}<P P_{s}
\end{array} \quad C F \in[-1,1] .\right.
$$

其中, $P P_{a}$ 为地震滑坡事件在因子分类 $a$ 中发生 的条件概率，即代表因子分类 $a$ 中存在的地震滑坡面 积百分比; $P P_{S}$ 为地震滑坡事件在整个研究区 $A$ 中发 生的先验概率, 可表示为整个研究区地震滑坡的面积 百分比, 为一定值 (许冲等, 2010a)。 $C F>0$ 表示地 震滑坡变形失稳的确定性高, 此单元为滑坡易发区; $C F<0$ 则表示此单元不易发生滑坡; $C F$ 接近 0 代表 事件发生的确定性难以确定, 即不能确定此单元是否 有利于发生滑坡。

陈晓利等 (2009) 通过搜集前人的研究成果和龙 陵地震滑坡的相关资料，对 1976 年龙陵地震诱发的 滑坡运用 $C F$ 法进行了影响因子的敏感性评价，确定 
了研究区内最有利于地震滑坡发生的数值区间。许冲 等 (2010a, 2010b) 运用 $C F$ 法，基于不同的影响因子 以及因子组合对 2008 年汶川地震滑坡进行了影响因 子敏感性研究以及易发性评价, 确定了滑坡因子的易 发区间以及危险性区划图。刘丽娜等（2014a）利用 $C F$ 法对 2013 年芦山地震滑坡的影响因子敏感性进行 了分析，对不同的地形因子间以及地质与地震因子间 的相互影响进行了探索研究。

\subsection{3. 信息量法}

信息量模型首先由晏同珍等（1989）基于信息论 引入了信息量法进行滑坡预测。具体到地震滑坡来 说, 通过对已发生地震滑坡进行分析, 把影响地震滑 坡各种因素的实测值转化为信息量值，用信息量值的 大小来衡量各影响因子与地震滑坡之间的作用程度

（阮沈勇和黄润秋, 2001）。该方法也是基于实际滑坡 数据得到因子的权重的一种二元统计方法, 避免了因 子权重赋值的主观性。有效样本数越多, 模型评价结 果越准确。但要注意评价因子选择的人为主观性。

信息量在实际计算时可用样本频率来估算条件 概率, 则各影响因素 $x_{i}$ 对地震滑坡事件 $A$ 提供的信息 量为:

$$
I\left(x_{i}, A\right)=\ln \frac{P\left(x_{i} \mid A\right)}{P\left(x_{i}\right)}=\ln \frac{N_{i} / N}{S_{i} / S} .
$$

那么假设参加评价的因子有 $n$ 个, 则单个评价单 元内总的信息量 $I_{i}$ :

$$
I_{i}=\sum_{i=1}^{n} I\left(x_{i}, \mathrm{~A}\right)=\sum_{i=1}^{n} \ln \frac{N_{i} / N}{S_{i} / S}=\sum_{i=1}^{n} \frac{N_{i} / S_{i}}{N / S} .
$$

其中, $I\left(x_{i}, A\right)$ 为滑坡分布条件下出现 $x_{i}$ 的概率, $P\left(x_{i}\right)$ 为研究区内出现 $x_{i}$ 的概率, $S$ 为研究区总单元 数, $N$ 为研究区滑坡所占的单元总数, $S_{i}$ 为研究区内 存在因素 $x_{i}$ 的单元个数, $N_{i}$ 为分布在因素 $x_{i}$ 内的特 定类别内的滑坡单元数。 $N_{i} / S_{i}$ 为滑坡在某影响因子 特定类别内的分布密度; $N / S$ 为研究区的滑坡面积百 分比, 为一定值（高克昌等, 2006）。

刘应辉（2009）运用信息量法对 2008 年汶川地 震震区都汶公路沿线崩塌滑坡灾害等进行了研究, 结 果表明该模型的评价效果较好。陶舒等 (2010) 等采 用信息量法与逻辑回归模型以 2008 年汶川地震后的 汶川县北部的地震次生滑坡灾害为例进行了敏感性 评价, 分别得到了 2 幅敏感性评估图, 结果表明信息 量法在极高敏感区内的评价精度也很高。牛全福等 （2011）运用信息量模型得到了 2010 年玉树地震诱 发滑坡的危险性区划图。Xu（2013）对 2013 年芦山
地震震区 3878 处地震滑坡运用基于专家知识和信息 量模型分别进行了地震滑坡危险性评价, 最终认为基 于信息量模型的芦山地震震区危险性评价精度较高。

\subsection{4.逻辑回归模型}

逻辑回归模型（Logistic Regression, LR）是通过 探索一个二值因变量和多个自变量之间形成的回归 关系，从而预测一定条件下某一事件的发生概率。逻 辑回归的优势在于其最优输出由最小二乘法给定, 且 进行统计分析时, 自变量可以是连续的, 也可以是离 散的（邢秋菊等, 2004; 刘艺梁等, 2010)。逻辑回归 模型作为一种多元统计方法, 具有操作方便、性能 稳定、计算过程受人为干扰小, 且利用回归系数较 好地体现了评价因子之间的相互关系。这种模型适 用于大区域地震滑坡危险性评价研究, 需要大量的 滑坡样本和不滑样本。该模型也是区域地质灾害研 究最常用的统计模型。

设 $P$ 为发生地震滑坡的概率, 取值范围 $[0,1]$, $(1-P)$ 为不发生地震滑坡的概率, 将 $P /(1-P)$ 取自然对 数 $\ln (P /(1-P))$, 记为 $\log$ it $\mathrm{P}$, 以 $P$ 为因变量, 建立 线性回归方程:

$$
\begin{gathered}
\log \text { it } P=\alpha+\beta_{1} x_{1}+\cdots+\beta_{m} x_{m} . \\
P=\frac{\exp \left(\alpha+\beta_{1} x_{1}+\cdots+\beta_{m} x_{m}\right)}{1+\exp \left(\alpha+\beta_{1} x_{1}+\cdots+\beta_{m} x_{m}\right)} .
\end{gathered}
$$

其中, $\alpha$ 为常数, $\beta_{1}$ 为回归系数。

Garcia-Rodriguez 等 (2008) 利用逻辑回归模型, 对 2001 年萨尔瓦多地震所诱发的滑坡灾害进行了危 险性评价, 认为地形粗䊁度和土地类型是影响该地区 滑坡发生的最重要的因素, 最后得到了该地区相对的 滑坡敏感性分布图。 $\mathrm{Su}$ 等（2010）基于 GIS 和逻辑 回归模型对 2008 年汶川地震后青川县的地震滑坡进 行了危险性区划， $65.3 \%$ 的滑坡分布在高和极高危险 性区域内。许冲和徐锡伟（2012c）针对 2010 年玉树 地震的地震滑坡进行了解译, 并运用逻辑回归模型建 立了滑坡危险性指数分布图，对比实际滑坡分布图与 滑坡危险性指数图得到模型的正确率达到了 $83.21 \%$ 。 $\mathrm{Xu}$ 等 (2013) 运用逻辑回归模型对 2008 年汶川地震 所诱发的近 20 万处滑坡进行了危险性区划，实际滑 坡分布与敏感指数图对比的结果表明, 模型的成功率 $83.751 \%$, 预测率 $86.930 \%$ 。李晓璇和马海建 (2013)、 文海家等 (2014) 分别基于逻辑回归模型对汶川地震 重灾区汶川县的地震滑坡进行了危险性评价, 李晓璇 和马海建等利用 998 处滑坡样本、998 处非滑坡样本 点基于坡度、断裂带、地形起伏度、岩性和烈度 5 个 因子进行分析, 认为 $72.4 \%$ 的灾害点位于高和极高危 险区; 文海家等利用 149 个滑坡样本点、751 个不滑 
样本点基于高程、坡度、坡位、坡向、岩性、微地貌 等 12 个因子进行分析,认为 $61.2 \%$ 的滑坡位于高和极 高危险区, 模型的正确率高达 94\%。Li 等（2013a） 对 2008 年汶川地震震区 37 个重灾县中的前 10 个县 的 43842 处地震滑坡运用逻辑回归方法进行了地震滑 坡危险性评价, 结果表明在占研究区总面积 3.9\%的高 和极高危险区内分布有 $73.3 \%$ 的滑坡, 模型的成功率 也达到了 $80 \%$ 。

\subsection{5. 人工神经网络}

人工神经网络 (Artifieial Neural Newtork, ANN), 是通过模拟人脑的思维, 把不同层的大量神经元连接 成一个复杂的网络, 用具有代表性的已知样本对神经
元之间的连接进行训练, 在不断地训练和检验的过程 中实现输出与目标的一致, 进而建立起不同输入层隐层-输出层之间的非线性映射关系并对未知样本进 行预测（Ermini 等, 2005; Chen 等, 2009)。其中, BP 神经网络（Back-Propagation Neural Network, 反向传 播神经网络）模型是目前应用最广泛的的一种神经网 络模型。对于地震滑坡危险性评价来说, 就是将不同 的影响因子和具有代表性的滑坡样本作为输入层, 通 过隐层处理后输出, 若输出结果与预测样本不符, 输 出误差将朝着误差最小的方向通过隐层向输入层传 递, 从而修正输入层与隐层以及隐层与输出结果的权 重和阈值, 通过反复训练, 最后得到与实际滑坡分布 一致的危险性评价模型, 用于对未发生滑坡区域进行 危险性区划。

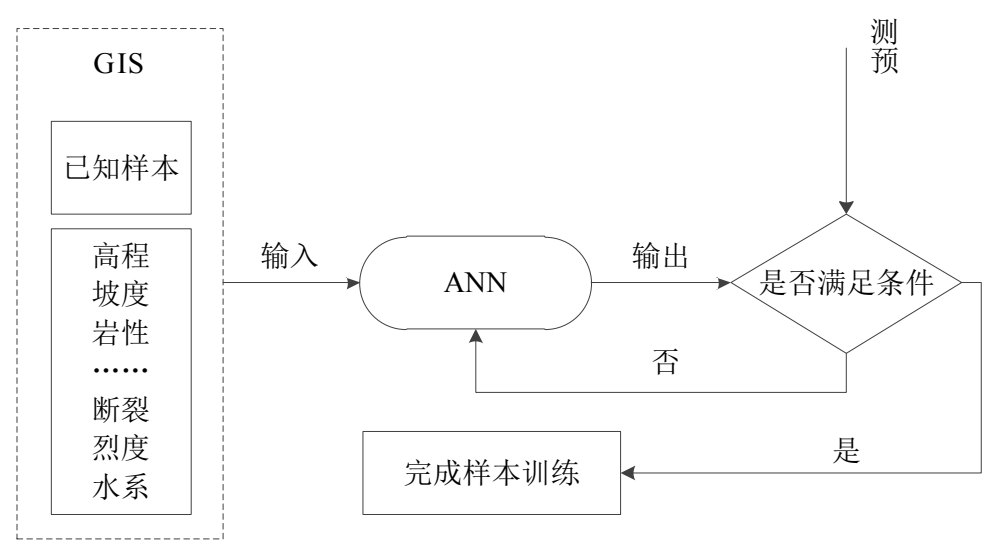

图 2.神经网络的基本原理

Fig. 2.Basic principles of the neural network.

Lee 和 Evangelista（2006）基于 GIS 和 BP 神经 网络针对 1990 年发生在菲律宾碧瑶市的地震所触发 的滑坡样本进行训练, 最后将所得神经网络模型和已 知滑坡进行验证对比, 模型预测的成功率达到了 93.2\%。陈晓利等（2006）利用径向基概率神经网络 自学习的特性, 以 1976 年龙陵地震触发的 32 个大型 滑坡和滑坡群所在的区域为研究区, 基于区内已知的 滑坡单元和未发生滑坡的单元为训练样本, 通过样本 的自主学习训练对待识别样本进行判断, 最终的识别 精度达到了 $89.9 \%$ 。García-Rodríguez 和 Malpica (2010) 基于人工神经网络法针对 2001 年 EI Salvador 地震所诱发的滑坡灾害以及影响边坡稳定性的各因 素进行了危险性评价, 其中有 $83 \%$ 的滑坡位于极高和 高危险区。许冲和徐锡伟 (2012a) 利用 BP 神经网络 以滑坡的质心位置和滑坡源区坡体高程最大位置为
训练样本对 2010 年玉树地震滑坡进行危险性区划, 最终模型的预测率都达到了 $81 \%$ 。 $\mathrm{Li}$ 等 (2012) 对 2008 年汶川地震后青川县的 855 处地震滑坡以及震前 的 473 处降雨滑坡运用 BP 神经网络模型进行了危险 性评价, 基于地震诱发滑坡的神经网络模型成功率为 $98 \%$ 。

人工神经网络虽然在工程领域得到了认可, 但当 滑坡数量和评价因子数量较多时, 计算量较大是十分 突出的问题; 并且人工神经网络对于输入层的优化存 在着黑箱操作问题, 很难解释和验证其反演结果。

\subsection{6. 支持向量机}

支持向量机（Support Vector Machines, SVM）由 Vapnik（2000）创建, 是一种基于统计的模型, 能够 
在样本较少的情况下, 通过核函数将线性不可分的数 据转换成高维空间的线性可分数据。基于结构风险最 小原则和求解受限二次型使得支持向量机法可以在 理论上获得最优解, 因此支持向量机在有效滑坡样本 和影响因子较少情况下, 可以获得较合理的危险性评 价结果。其中, 支持向量机模型的重要组成部分是核 函数。常见的核函数主要有 (Cristianini 和 Scholkopf, 2002；戴福初等, 2008; 谭龙等, 2014)：

线性核函数 (Linear):

$$
K\left(x_{i}, x_{j}\right)=x_{i}^{T} x_{j} .
$$

多项式核函数（Polynomial）:

$$
K\left(x_{i}, x_{j}\right)=\left(\gamma x_{i}^{T} x_{j}+r\right)^{d}, \gamma>0 .
$$

径向基核函数（Radial basis function）:

$$
K\left(x_{i}, x_{j}\right)=e^{-\gamma\left(x_{i}-x_{j}\right)^{2}} .
$$

$\mathrm{S}$ 形核函数 (Sigmoid):

$$
K\left(x_{i}, x_{j}\right)=\tan \left(\gamma x_{i}^{T} x_{j}+r\right) .
$$

$\gamma 、 r 、 d$ 是核函数的参数, 计算时需要人工输 入。其中，基于径向基核函数的支持向量机模型得到 的评价结果效果最好。

$\mathrm{Xu}$ 和 $\mathrm{Xu}$ （2012）、许冲和徐锡伟（2012）基于 不同的核函数 (线性核函数、多项式核函数、径向基 核函数和 S 形核函数）运用支持向量机法对 2010 年 玉树地震所诱发的 2036 处滑坡进行了空间预测和危 险性分级, 并指出径向基核函数是最适于该地区地震 滑坡空间预测的模型，其模型正确率达 $84.16 \%$ 。Xu 等 (2012a) 以 2008 年汶川震区涪江流域的地震滑坡 为研究对象, 基于支持向量机法, 选用了线性核函数、 多项式核函数和径向基核函数进行了滑坡敏感性区 划, 模型的成功率和预测率都在 $70 \%$ 以上, 且径向基 函数的向量机模型成功率和预测率最高。

\subsubsection{Newmark 位移模型}

Newmark 位移模型假设地震触发的滑坡体为刚 性块体, 基于极限平衡理论, 通过计算滑体在地震荷 载作用下沿着危险滑动面所发生的永久位移量来评 价斜坡的稳定性，而永久位移可以通过对地震荷载加 速度和滑体滑动的临界加速度的差值进行积分得到, 所以该方法的重点转移到了求取滑坡体本身的临界 加速度和安全系数上。临界加速度和安全系数可以通 过岩土体的力学性质 (有效内聚力、有效内摩擦角、 岩土体重度等) 以及坡度等计算得到, 这就使得该方 法在地震滑坡危险性评价中得到了普及（Newmark, 1965; Jibson 等, 2000; 王涛等, 2013)。

正是由于 Newmark 位移方法利用临界加速度 或 Newmark 位移等来评价地震滑坡的危险性, 所 以该方法不需要滑坡样本数据, 仅根据震区的岩土 体强度和坡度来判断危险区域。但这种方法忽略了 区域地质体的局部差异性和复杂性以及坡体的规 模。因此当缺乏较详细区域岩土体的实测强度参 数、地震动参数等资料时, Newmark 位移方法的精 度还有待提高。

Refice 和 Capolongo (2002) 基于简化的 Newmark 方法对意大利南部 Irpinian 地区的地震滑坡进行了危 险性评价, 与其他评价方法的结果基本一致。Jibson 等 (2000) 对 1994 年 Northridge 地震所诱发的滑坡 利用 Newmark 永久位移法进行了分析, 得到了滑坡 的危险性区划图。陈晓利等 (2013) 利用 Newmark 模型对 2013 年芦山震区烈度大于 VII 的区域内的地 震滑坡进行了空间分布预测, 虽然预测结果与实际滑 坡分布情况有一定的出入, 但该方法仍表现出了较高 的可行性。

\section{2. 知识驱动方法}

层次分析法 (AnalyticHierarchy Process, AHP) 属 于常用的知识驱动模型, 是由美国匹兹堡大学教授 Saaty 在 20 世纪 70 年代中期提出的面向多因素、多 层次、多目标的复杂系统和难于完全用定量的方法来 分析与决策的复杂系统工程问题的处理方法, 其思想 是通过构造判断矩阵对比两两影响因子, 确定诸因子 的相对重要性（Saaty, 1988; 陈晓利, 2007)。判断矩 阵标度及含义见表 1 。 
表 1.层次分析法判断矩阵的标度和含义

Table.1.The scale and the meanings of the AHP matrix.

\begin{tabular}{|c|c|}
\hline 标度值 & 含义 \\
\hline 1 & 表示两个因素相比, 具有相同重要性 \\
\hline 3 & 表示两个因素相比, 前者比后者稍重要 \\
\hline 5 & 表示两个因素相比, 前者比后者明显重要 \\
\hline 7 & 表示两个因素相比, 前者比后者强烈重要 \\
\hline 9 & 表示两个因素相比, 前者比后者极端重要 \\
\hline $2,6,8$ & 表示上述相邻判断的中间值 \\
\hline 倒数 & 与上述影响情况相反 \\
\hline
\end{tabular}

AHP 法作为一种多指标分析方法, 其基本操作流 程为由专家将影响地震滑坡发生的因素进行简单比 较和计算, 得到每个因子合理的权重, 然后进行危险 性评价。这是一种定性和定量相结合的方法。虽然带 有一定的主观性, 但是通过比较影响因子得到的权重 避免了较不合理的情况。这种方法不需要选择滑坡样 本, 仅依靠专家知识和影响因子基础数据即可实现, 操作方便快捷, 精度较高。

许冲等（2009）利用 AHP 法对 2008 年汶川地震 震区的 48007 个滑坡进行了危险性评价, 模型成功率 达到了 79.656\%。杜军和杨青华 (2009)、张建强等 （2009）基于 AHP 模型, 确定了 2008 年汶川震区汶 川县的地震次生地质灾害各评价因子的权重, 并进行 了风险性评估, 结果与实际灾害分布一致。Tang 等 （2009）基于专家知识, 利用 AHP 法获取 2008 年汶 川地震震区青川县地震滑坡影响因子的权重并进行 了该地区地震滑坡危险性评价, 然后又将该评价模型 应用于北川县城地震滑坡进行危险性评价, 都得到了 较好的结果。刘丽娜等 (2014b) 使用 AHP 法采用多 名专家估计方案对 2013 年芦山地震震区一个矩形区 域内的滑坡进行了危险性评价, 模型的成功率高达 $85.41 \%$ 。

\section{5. 实例分析}

以 2008 年汶川地震震区嘉陵江涪江一个支流流 域为研究区, 通过实地调查和对覆盖整个研究区的 $1 \mathrm{~m}$ 分辨率的航片进行目视解译, 得到地震诱发滑坡 944 处。选取了坡度、坡向、高程、曲率、距河流的 距离、地形湿度指数 (TWI)、距主干道路的距离以 及距地表破裂带的距离、PGA 和岩性等 10 个影响地 震滑坡发生的因素，分别基于二元统计模型、逻辑回 归模型、人工神经网络、线性核函数向量机模型、多 项式核函数向量机模型、径向基核函数向量机模型进
行了危险性评价, 最终得到了研究区内的地震滑坡危 险性分区图 (图 3)（Xu 等, 2012c)。

运用实际滑坡数量百分比累加-危险性面积百分 比累积曲线的曲线下面积方法对这 6 种模型评价结果 的成功率和预测率进行定量检验。各模型的曲线下面 积见图 4。从图中可以看出, 模型的成功率和预测率 由高到低依次为: 逻辑回归模型、基于径向基函数的 支持向量机模型、人工神经网络、基于多项式函数的 支持向量机模型、二元统计模型、线性函数向量机模 型。其中逻辑回归模型的成功率和预测率均最高 (AUC 分别为 $80.34 \%$ 和 $80.27 \%$ )。

分析结果表明, 在该研究区内, 基于逻辑回归模 型的地震滑坡危险性评价结果最好, 其次为基于径向 基函数的支持向量机模型。
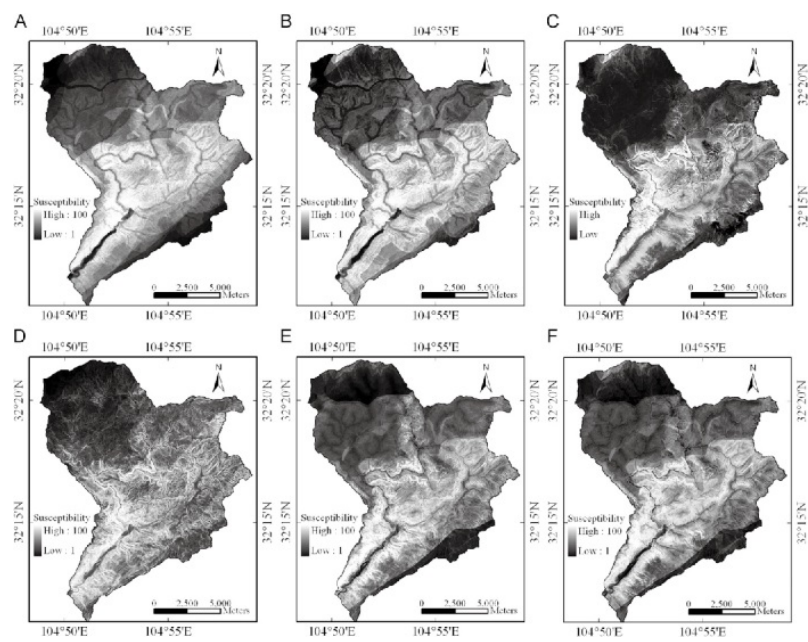

图 3 涪江流域地震滑坡危险性分区图（Xu 等, 2012c)

Fig.3. Landslide susceptibility mapsbased on different models in Fu River basin. (A)二元统计模型; (B) 逻辑回归模 型; (C) 人工神经网络; (D) 线性核函数向量机模型; (E) 多 项式核函数向量机模型; (F) 径向基核函数向量机模型。 

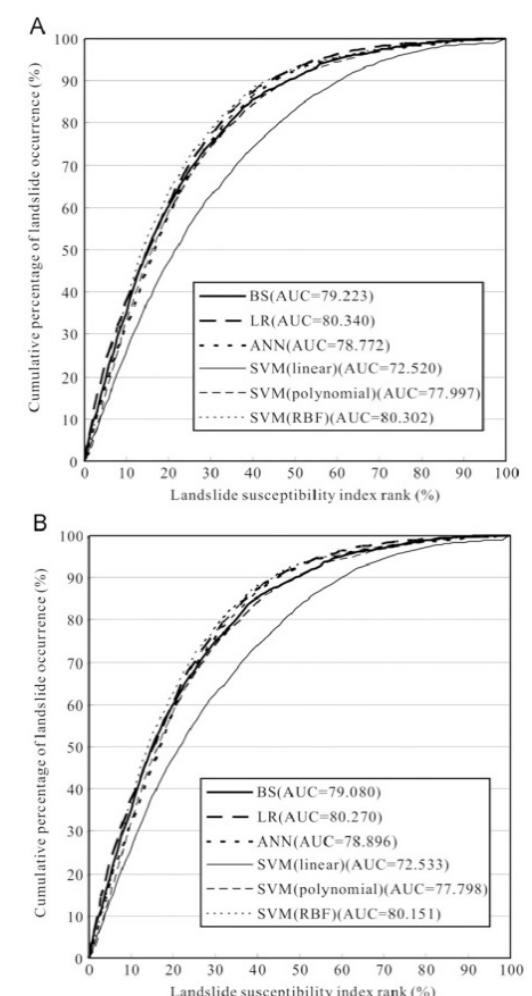

图 4.滑坡危险性评价结果评价曲线 ( $\mathrm{Xu}$ 等, 2012c)

Fig.4. Area under curve (AUC) representing the success rate and prediction rate of the models used.

(A) 成功率曲线; (B) 预测率曲线。

\section{6. 结论与展望}

\section{1.结论}

（1）随着 GIS 的发展, 特别是其空间分析功能 的完善, 通过结合 GIS 和各种评价方法, 地震滑坡危 险性评价变得更加便捷, 评价精度和效率也得到了很 大程度的提高。利用 GIS 的制图功能, 也可做出各种 危险性区划图件，方便灾区支援和减灾工作。

（2）地震滑坡编目是地震滑坡危险性评价的数 据基础。通过各种评价模型分析各影响因子对地震滑 坡的贡献，从而进行地震滑坡危险性区划。常用的地 震滑坡危险性评价的模型有证据权重模型、确定性系 数法 $(C F)$ 、信息量法、逻辑回归模型 $(L R)$ 、人工 神经网络 $(A N N)$ 、支持向量机法 $(S V M)$ 、 Newmark 位移模型和层次分析法 (AHP) 等。这些方法各有利 弊：数据驱动方法中，证据权重模型、确定性系数法 和信息量法均基于实际滑坡数据, 避免了不滑样本选 取的随意性和因子赋值的主观性, 适用于存在大量有 效滑坡样本的区域, 在评价时要尽量将影响因子考虑
全面。逻辑回归模型也适用于存在大量滑坡样本的区 域, 但是对于不滑样本的选取没有统一的标准, 这往 往会影响评价的结果。人工神经网络和支持向量机模 型由于处理过程的复杂性, 数据量较大, 适用于小区 域地震滑坡危险性评价。Newmark 位移法将岩土体的 力学性质引入区域滑坡危险性评价中，仅根据震区的 岩土体强度和坡度来判断危险区域, 忽略了区域地质 体的局部差异性和复杂性以及坡体的规模。因此只适 用于具备较详细区域岩土体的实测强度参数、地震动 参数等资料的小区域地震滑坡危险性评价。文中以 2008 年汶川地震震区嘉陵江涪江一个支流流域为例, 基于二元统计模型、逻辑回归模型、人工神经网络、 线性核函数向量机模型、多项式核函数向量机模型、 径向基核函数向量机模型，对研究区内的 944 个地震 滑坡进行了危险性评价。结果表明, 基于逻辑回归模 型的地震滑坡危险性评价结果最适用于本研究区。

\section{2. 展望}

（1）虽然地震滑坡危险性评价已经进入定量阶 段, 但由于每种评价方法自身的适用性和局限性, 加 之地震的预测十分困难，从而造成了危险性评价因子 和评价结果的各种不确定性和模糊性。另外, 由于地 震滑坡形成的力学机理和环境条件十分复杂, 所以目 前地震滑坡危险性评价的方法仍有待改进, 必要时可 以综合两种或多种评价模型进行评价。

（2）较好的评价结果必须建立在对历史地震滑 坡研究较充分的基础上, 但在进行地震滑坡解译的时 候, 震后第一时间的遥感影像并不容易获得, 影像的 质量、分辨率和云量等都影响编目结果的准确性。

（3）选取评价因子以及对评价因子赋权重时, 需考虑地震滑坡影响因子选取的合理性、全面性以及 最优因素组合，尽量降低外界因素对评价结果的影 响, 使评价结果更加客观。

（4）传统的地震滑坡统计分析方法仅考虑了影 响滑坡发生的表层因素, 而这些因素大多是静态的, 这就很可能忽略了对滑坡发生的起关键作用的因素， 比如断裂深部的形态以及坡体内含水量等对滑坡发 生的影响。因此，进行危险性评价时要基于滑坡所处 的地质背景，同时也要关注对滑坡自身形态以及滑坡 物源区、流通区以及堆积区的形态和规模等与各影响 因素之间的关系, 并加强对区域地震滑坡危险性评价 的物理或数值模拟研究。

\section{致谢}

本文由国家自然科学基金（41472202）资助。 


\section{参考文献}

D. K. Keefer, Investigating landslides caused by earthquakes-a historical review,Surveys in Geophysics, 23(6) (2002) 473-510.

W. L. Li, R. Q. Huang, X. J. Pei and X. C. Zhang, Historical co-seismic landslide inventory with Google Earth: A case study of 1920 Haiyuan Earthquake, China,Global View of Engineering Geology and the Environment. Boca Raton: CRC Press, (2013a) 179-184.

吴玲, 基于 GIS 和 RS 什邡市山区地震滑坡主要影响因子 的研究. (四川农业大学, 四川, 2012).

E. L. Harp and R. W. Jibson, Landslides triggered by the 1994 Northridge, California, earthquake,Bulletin of the Seismological Society of America, 86(1B) (1996) S319-S332.

C.T. Lee, Re-Evaluation of Factors Controlling Landslides Triggered by the 1999 Chi-Chi Earthquake, in Earthquake-Induced Landslides (Springer, 2013), pp. 213-224.

殷跃平, 汶川八级地震滑坡特征分析, 工程地质学报, 17(01) (2009) 29-38.

E. Calais, A. Freed, G. Mattioli, F. Amelung, S. Jónsson, P. Jansma, S.H. Hong, T. Dixon, C. Prépetit and R. Momplaisir, Transpressional rupture of an unmapped fault during the 2010 Haiti earthquake,Nature Geoscience, 3(11) (2010) 794-799.

许冲, 2010 年海地太子港地震触发滑坡危险性区划和合理 性检验，科技导报, 31(12) (2013a) 42-47.

许冲, 徐锡伟, 戴福初, 王彦颖, 2010 年 4 月 14 日玉树 地震滑坡空间分布与控制变量分析，工程地质学报， 19(4) (2011) 505-510.

许冲, 2013 年芦山地震 Ms7.0 级滑坡易发性快速评价方法. 科技导报, 31(28/29) (2013b) 15-23.

许冲，徐锡伟，郑文俊，闵伟，任治坤 and 李志强，2013 年甘肃岷县漳县 6.6 级地震触发滑坡及其构造分析, 地震地质, 35(3) (2013) 616-626.

陈晓利, 祁生文,叶洪, 基于 GIS 的地震滑坡危险性的模糊 综合评价研究，北京大学学报: 自然科学版, 44(3) (2008) 434-438.

庄建琦, 崔鹏, 葛永刚, 朱颖彦, 刘应辉, 裴来政, “ $5 \cdot 12$ ” 汶川地震崩塌滑坡危险性评价一一以都汶公路沿线为 例，岩石力学与工程学报, 29(增 2) (2010) 3735-3742.

葛华，陈启国，王德伟，地震滑坡危险性评价及编图一一以 映秀震中区为例, 中国地质, 40(2) (2013) 644-652.

E. L. Harp, D. K. Keefer, H. P. Sato and H. Yagi, Landslide inventories: the essential part of seismic landslide hazard analyses. Engineering Geology, 122(1) (2011) 9-21.

许冲, 徐锡伟, 21 世纪初几次大地震事件触发滑坡基础数 据建设，地震地质, 36(1) (2014) 90-104.

C. Xu, Preparation of earthquake-triggered landslide inventory maps using remote sensing and GIS technologies: Principles and case studies, Geoscience Frontiers, 6(6) (2015) 825-836

C. Xu and X. W. Xu, Statistical analysis of landslides caused by the Mw 6.9 Yushu, China, earthquake of April 14, 2010,Natural Hazards, 72(2) (2014) 871-893.
C. Xu, J. B. H. Shyu and X. W. Xu, Landslides triggered by the 12 January 2010 Port-au-Prince, Haiti, $\mathrm{Mw}=7.0$ earthquake: visual interpretation, inventory compiling, and spatial distribution statistical analysis,Natural Hazards and Earth System Sciences, 14(7) (2014a) 1789-1818.

C. Xu, X. W. Xu and J. B. H. Shyu, Database and spatial distribution of landslides triggered by the Lushan, China Mw 6.6 earthquake of 20 April 2013,Geomorphology, 248((2015a) 77-92.

C. Xu, X. Xu, J. B. H. Shyu, M. Gao, X. Tan, Y. Ran and W. Zheng, Landslides triggered by the 20 April 2013 Lushan, China, Mw 6.6 earthquake from field investigations and preliminary analyses,Landslides, 12(2) (2015b) 365-385.

C. Xu, X. W. Xu, J. B. H. Shyu, W. J. Zheng and W. Min, Landslides triggered by the 22 July 2013 Minxian-Zhangxian, China, Mw 5.9 earthquake: Inventory compiling and spatial distribution analysis,Journal of Asian Earth Sciences, 92(2014b) 125-142.

许冲, 徐锡伟, 沈玲玲, 窦帅, 吴赛儿, 田颖颖, 李西, 2014 年鲁甸 Ms 6.5 地震触发滑坡编录及其对一些地震参数 的指示，地震地质, 36(4) (2014) 1186-1203.

C. Xu, X. Xu, X. Yao and F. Dai, Three (nearly) complete inventories of landslides triggered by the May 12, 2008 Wenchuan Mw 7.9 earthquake of China and their spatial distribution statistical analysis,Landslides, 11(3) (2014c) 441-461.

谢洪斌，杨雪，罗真富，模糊证据权法在地震滑坡危险度区 划中的应用，中国安全科学学报, 21(8) (2011) 164-170.

许冲, 2008 年汶川地震前的中国大陆地震滑坡研究, 科技 导报, 32(16) (2014) 63-77.

S. Lee and J. Choi, Landslide susceptibility mapping using GIS and the weight-of-evidence model,International Journal of Geographical Information Science, 18(8) (2004) 789-814.

王志旺, 李端有, 王湘桂, 证据权法在滑坡危险度区划研究 中的应用，岩土工程学报, 29(8) (2007) 1268-1273.

R. K. Dahal, S. Hasegawa, A. Nonomura, M. Yamanaka, S. Dhakal and P. Paudyal, Predictive modelling of rainfall-induced landslide hazard in the Lesser Himalaya of Nepal based on weights-of. Geomorphology, 102(3) (2008) 496-510.

赵艳南，牛瑞卿，基于证据权法的滑坡危险性区划探索，地 理与地理信息科学, 26(6) (2010) 19-23.

C. Xu, X. Xu, Y. H. Lee, X. Tan, G. Yu and F. Dai, The 2010 Yushu earthquake triggered landslide hazard mapping using GIS and weight of evidence modeling,Environmental Earth Sciences, 66(6) (2012a) 1603-1616.

C. Xu, X. W. Xu, F. C. Dai, J. Z. Xiao, X. B. Tan and R. M. Yuan, Landslide hazard mapping using GIS and weight of evidence model in Qingshui River watershed of 2008 Wenchuan earthquake struck region,Journal of Earth Science, 23(1) (2012b) 97-120.

张艳玲，南征兵，周平根，利用证据权法实现滑坡易发性区 划，水文地质工程地质，39(2) (2012) 121-125.

E. H. Shortliffe and B. G. Buchanan, A model of inexact reasoning in medicine. Mathematical biosciences, 23(3) (1975) 351-379.

D. Heckerman, Probabilistic interpretations for MYCIN's 
certainty factors, arXiv preprint arXiv:1304.3419, (2013).

兰恒星，伍法权，王思敬，基于 GIS 的滑坡 CF 多元回归模 型及其应用，山地学报, 20(06) (2002) 732-737.

兰恒星, 王苓涓, 周成虎，云南小江流域滑坡关键影响因子 研究. 中国地质灾害与防治学报, 40(01) (2003) 103-109.

许冲，戴福初，姚金金，陈剑，涂新斌，曹琰波，肖建章，基于 GIS 的汶川地震滑坡灾害影响因子确定性系数分析，岩 石力学与工程学报, 29(增 1) (2010a) 2972-2981.

陈晓利，手洪流，祁生文，1976 年龙陵地震诱发滑坡的影响 因子敏感性分析, 北京大学学报(自然科学版), 45(1) (2009) 104-110

许冲, 戴福初, 姚釒金, 赵洲, 肖建章, 基于 GIS 与确定性系 数分析方法的汶川地震滑坡易发性评价, 工程地质学报, 18(1) (2010b) 15-26.

刘丽娜, 许冲, 陈剑, GIS 支持下基于 CF 方法的 2013 年 芦山地震滑坡因子敏感性分析，工程地质学报，22(6) (2014a) 1176-1186

晏同珍, 伍法权, 殷坤龙, 滑坡系统静动态规律及斜坡不稳 定性空时定量预测，地球科学-中国地质大学学报，14(2) (1989) 117-133.

阮沈勇，黄润秋，基于 GIS 的信息量法模型在地质灾害危险 性区划中的应用，成都理工学院学报，28(01) (2001) 89-92.

高克昌，崔鹏，赵纯勇，韦方强，基于地理信息系统和信息 量模型的滑坡危险性评价-—以重庆万州为例，岩石力 学与工程学报, 25(5) (2006) 991-996.

刘应辉, 汶川地震区都汶公路沿线崩塌滑坡灾害特征与评 价. (兰州大学, 兰州, 2009).

陶舒, 胡德勇, 赵文吉, 范一大, 王志恒, 基于信息量与逻 辑回归模型的次生滑坡灾害敏感性评价——以汶川县 北部为例，地理研究，29(09) (2010) 1594-1605.

牛全福, 程维明, 兰恒星, 刘勇, 颉耀文, 基于信息量模型 的玉树地震次生地质灾害危险性评价，山地学报, 29(02) (2011) 243-249.

C. $\mathrm{Xu}$, Assessment of earthquake-triggered landslide susceptibility based on expert knowledge and information value methods: a case study of the 20 April 2013 Lushan, China Mw6. 6 earthquake,Disaster Advances, 6(13) (2013) 119-130.

邢秋菊, 赵纯勇, 高克昌, 郭跃, 基于 GIS 的滑坡危险性逻 辑回归评价研究, 地理与地理信息科学, 20(03) (2004) 49-51.

刘艺梁，殷坤龙，刘斌，逻辑回归和人工神经网络模型在滑 坡灾害空间预测中的应用，水文地质工程地质，37(5) (2010) 92-96.

M. J. Garcia-Rodriguez, J. A. Malpica, B. Benito and M. Diaz, Susceptibility assessment of earthquake-triggered landslides in El Salvador using logistic regression, Geomorphology, 95(3-4) (2008) 172-191.

F. Su, P. Cui, J. Zhang and L. Xiang, Susceptibility assessment of landslides caused by the wenchuan earthquake using a logistic regression model.,Journal of Mountain Science, 7(3) (2010) 234-245.

许冲, 徐锡伟, 逻辑回归模型在玉树地震滑坡危险性评价 中的应用与检验, 工程地质学报, 20(3) (2012a) 326-333.

C. Xu, X. Xu, F. Dai, Z. Wu, H. He, F. Shi, X. Wu and S. Xu, Application of an incomplete landslide inventory, logistic regression model and its validation for landslide susceptibility mapping related to the May 12, 2008 Wenchuan earthquake of China,Natural Hazards, 68(2) (2013) 883-900.

李晓璇，马海建，基于 Logistic 模型的地震次生崩滑危险 性评价——以汶川县为例, 地震, 33(2) (2013) 63-70.

文海家，胡东萍，王桂林，汶川县地震滑坡易发性 LR 与 $\mathrm{NN}$ 评价比较研究, 土大工程学报，47(增 1) (2014) 17-23.

W. L. Li, R. Q. Huang, C. Tang, Q. Xu and C. van WESTEN, Co-seismic landslide inventory and susceptibility mapping in the 2008 Wenchuan earthquake disaster area, China,Journal of Mountain Science, 10(3) (2013b) 339-354.

L. Ermini, F. Catani and N. Casagli, Artificial neural networks applied to landslide susceptibility assessment,Geomorphology, 66(1) (2005) 327-343.

C. H. Chen, C. C. Ke and C. L. Wang, A back-propagation network for the assessment of susceptibility to rock slope failure in the eastern portion of the Southern Cross-Island Highway in Taiwan,Environmental Geology, 57(4) (2009) 723-733.

S. Lee and D. Evangelista, Earthquake-induced landslide-susceptibility mapping using an artificial neural network,Natural Hazards and Earth System Science, 6(5) (2006) 687-695.

陈晓利，赵健，叶洪，应用径向基概率神经网络研究地震滑 坡, 地震地质, 28(3) (2006) 430-440.

M. García-Rodríguez and J. Malpica, Assessment of earthquake-triggered landslide susceptibility in El Salvador based on an Artificial Neural Network model,Natural Hazards and Earth System Science, 10(6) (2010) 1307-1315.

许冲, 徐锡伟, 基于 GIS 与 ANN 模型的地震滑坡易发性 区划，地质科技情报，31(3) (2012b) 116-121.

Y. Li, G. Chen, C. Tang, G. Zhou and L. Zheng, Rainfall and earthquake-induced landslide susceptibility assessment using GIS and Artificial Neural Network,Natural Hazards and Earth System Sciences, 12(8) (2012) 2719-2729.

V. Vapnik (eds), The nature of statistical learning theory (Springer Science \& Business Media, 2000).

N. Cristianini and B. Scholkopf, Support vector machines and kernel methods: the new generation of learning machines, Ai Magazine, 23(3) (2002) 31-41.

戴福初，姚釒金，谭国焕，滑坡灾害空间预测支持向量机模型 及其应用，地学前缘, 14(6) (2008) 153-159.

谭龙，陈冠，王思源，孟兴民，逻辑回归与支持向量机模型 在滑坡敏感性评价中的应用，工程地质学报，22(01) (2014) 56-63.

C. Xu and X. W. Xu, Spatial prediction models for seismic landslides based on support vector machine and varied kernel functions: A case study of the 14 April 2010 Yushu earthquake in China,Chinese Journal of Geophysics, 55(6) (2012) 666-679.

许冲, 徐锡伟, 基于不同核函数的 2010 年玉树地震滑坡 空间预测模型研究，地球物理学报，55(9) (2012c) 2994-3005. 
C. Xu, X. Xu, F. Dai and A. K. Saraf, Comparison of different models for susceptibility mapping of earthquake triggered landslides related with the 2008 Wenchuan earthquake in China, Computers \& Geosciences, 46(2012c) 317-329.

N. M. Newmark, Effects of earthquakes on dams and embankments, Geotechnique, 15(2) (1965) 139-160.

R. W. Jibson, E. L. Harp and J. A. Michael, A method for producing digital probabilistic seismic landslide hazard maps,Engineering Geology, 58(3) (2000) 271-289.

王涛，吴树仁，石菊松，辛鹏，基于简化 Newmark 位移模 型的区域地震滑坡危险性快速评估一一以汶川 M S8. 0 级地震为例, 工程地质学报, 21(1) (2013) 16-24.

A. Refice and D. Capolongo, Probabilistic modeling of uncertainties in earthquake-induced landslide hazard assessment,Computers \& Geosciences, 28(6) (2002) 735-749.

陈晓利, 袁仁茂,d 属露, Newmark 方法在芦山地震诱发滑 坡分布预测研究中的应用，地震地质，35(3) (2013) 661-670.

T. L. Saaty (eds), What is the analytic hierarchy process? (Springer, 1988).

陈晓利, 人工智能在地震滑坡危险性评价中的应用. (中国 地震局地质研究所, 北京, 2007).

许冲, 戴福初, 姚釒金, 陈剑, 涂新斌, 孙瑜, 王志一, GIS 支 持下基于层次分析法的汶川地震区滑坡易发性评价，岩 石力学与工程学报, 28(增 2) (2009) 3978-3985.

杜军 and 杨青华, 基于 GIS 与 AHP 耦合的汶川震后次生地 质灾害风险评估。中国水土保持, 11) (2009) 14-16.

张建强，范建容，严冬，郭芬芬，苏凤环，地震诱发崩塌滑 坡敏感性评价一一以北川县为例，四川大学学报(工程 科学版), 41(03) (2009) 140-145.

C. Tang, J. Zhu and J. Liang, Emergency assessment of seismic landslide susceptibility: a case study of the 2008 Wenchuan earthquake affected area,Earthquake Engineering and Engineering Vibration, 8(2) (2009) 207-217.

刘丽娜，许冲，徐锡伟，陈剑，GIS 支持下基于 AHP 方法的 2013 年芦山地震区滑坡危险性评价，灾害学，29(04) (2014b) 183-191. 